Meta

Journal des traducteurs

Translators' Journal

\title{
Terminologie, langue et discours juridiques. Sens et signification du langage du droit
}

\section{Jean-Claude Gémar}

Volume 36, numéro 1, mars 1991

La terminologie dans le monde : orientations et recherches

URI : https://id.erudit.org/iderudit/002843ar

Aller au sommaire du numéro

Éditeur(s)

Les Presses de l'Université de Montréal

ISSN

0026-0452 (imprimé)

Découvrir la revue

Citer cet article

Gémar, J.-C. (1991). Terminologie, langue et discours juridiques. Sens et signification du langage du droit. Meta, 36(1), 275-283. d'utilisation que vous pouvez consulter en ligne. 


\section{TERMINOLOGIE, LANGUE ET DISCOURS JURIDIQUES. SENS ET SIGNIFICATION DU LANGAGE DU DROIT*}

JEAN-Claude GÉmar

Université de Montréal, Montréal, Canada

Des quatre éléments généralement reconnus comme constitutifs du langage du droit: sens, syntaxe, lexique et style, c'est le premier qui reste le plus impénétrable. Les trois autres sont bien connus et leur statut suffisamment établi pour faire ici l'objet d'une nouvelle étude. Le présent article portera donc uniquement sur le problème du sens, lequel sera envisagé dans sa généralité tout d'abord avant d'être considéré dans l'aspect particulier caractérisant l'expression (linguistique) du Droit.

\section{LANGUE JURIDIQUE ET LANGUE GÉNÉRALE}

Parler de «langue juridique» pour désigner le mode d'expression linguistique particulier aux juristes revient à dire que ces derniers empruntent, pour exprimer le droit, des voies différentes de celles du commun des mortels (le profane), lequel ne s'exprimerait que par le canal de la langue générale ou commune.

Autrement dit, il y aurait les mots, essentiellement généraux ou «simples» par vocation sinon par essence, par opposition à d'autres, les termes, qui auraient pour seule fonction de caractériser un type particulier de discours, puisqu'ils lui confèreraient, de par leur charge plus ou moins grande de sens spécialisé, une signification singulière voire univoque propre à un champ d'activité donné.

Or, l'ensemble des termes d'un domaine constitue, selon la classification qu'en a proposé Darbelnet $(1979: 26 ; 1982: 51)$ et que je reprends en la développant, une nomenclature (la terminologie d'un domaine particulier), noyau dur des notions fondamentales particulières à celui-ci, à partir de laquelle se réaliserait le discours spécialisé la caractérisant, que l'on qualifie aussi parfois de «langue de spécialité». Une langue de spécialité (LS) serait donc composée d'un noyau de termes qui, associés à des cooccurrents précis (le vocabulaire de soutien) et à des mots de la langue dite générale (le vocabulaire général), réaliseraient en trois étapes successives, sous l'impulsion d'une force centrifuge, par ondes se déplaçant dans le sens particulier $\Rightarrow$ général, le texte d'un discours spécialisé donné, par addition d'éléments de sens partiel, du plus complexe au plus simple, dont la somme apporterait au texte la plénitude de sa signification. $\mathrm{Ce}$ cheminement théorique pourrait être représenté par la formule

$\frac{\text { TERME }}{\text { COOCCURRENT(s) + Vogén }}=\mathbf{L S}$


Appliquons-la à un cas concret :

1. TERME = délit

2. Cooccurrent $=$ commettre [plutôt que : «faire», «entreprendre» ou «former»]

3. Vogén = quiconque, un, sera puni, sévèrement
Soit:
(1)
(2)
(3) (4)
(5) (6)

On voit par cet exemple simple que le message, pour être totalement exprimé - et sans doute compris -, doit contenir les trois niveaux complémentaires de termes, mots ou prédicats énoncés. L'absence d'un des éléments contribuant à la pleine signification du message déséquilibrerait la phrase et en diviserait d'autant le sens, l'amputant plus ou moins gravement selon que le segment omis sera

$\begin{array}{llcr}\text { un terme : } & \text { Quiconque commet un ... sera puni sévèrement } \\ \text { un cooccurrent : } & \text { Quiconque } & \ldots & \text { un délit sera puni sévèrement } \\ \text { ou un mot du Vogén: } & \ldots & \text { commet un délit sera puni sévèrement }\end{array}$

Quelle que soit la réduction partielle du sens considérée $(-1,-2,-3,-7$ ou -4$)$, il reste que la réalisation du discours, et donc l'expression du sens, passe obligatoirement par l'agrégat de ces trois éléments constitutifs du sens élémentaire ou minimal d'un texte, celui de la phrase donnée en exemple, et que tout lecteur, juriste ou non, comprendra sans coup férir. Cette phrase ne prendra toute sa signification que dans le contexte où elle se situe, lequel la liera à un sens particulier donné (telle loi, dans telle situation), et non plus libre ou général, ou encore élémentaire, comme dans l'exemple présenté. De cela il ressort que si le sens est bien affaire de signes linguistiques - ce qui compose les «mots», qu'il s'agisse de prédicats, de termes ou que sais-je encore -, la signification, elle, est extra-linguistique. En d'autres termes, les signes concourent à construire un sens lorsqu'ils sont agencés de façon à être reconnus comme signifiants par l'œil et l'esprit humains, ils contribuent à réaliser le texte à partir duquel le lecteur extraira la signification du message lu. L'élémentaire est d'ordre linguistique, l'essentiel de nature extra-linguistique.

En schématisant, la structure du discours spécialisé pourrait être idéalement représentée par une série de cercles concentriques dont le premier, au centre, serait le noyau dur (la nomenclature, soit la charge notionnelle des termes, en nombre limité); le cercle suivant, plus large, contiendrait le vocabulaire de soutien (les cooccurrents du domaine), à la fois moins nombreux mais aux possibilités d'association par agrégat naturel néanmoins bien supérieures; le troisième cercle, le plus éloigné, symboliserait les éléments aléatoires de la langue que sont les «mots» (articles, pronoms, verbes, adjectifs, ...), du vocabulaire général, en nombre quasi illimité, auxquels il faut recourir pour construire une phrase conforme aux normes de la syntaxe de la langue considérée. 


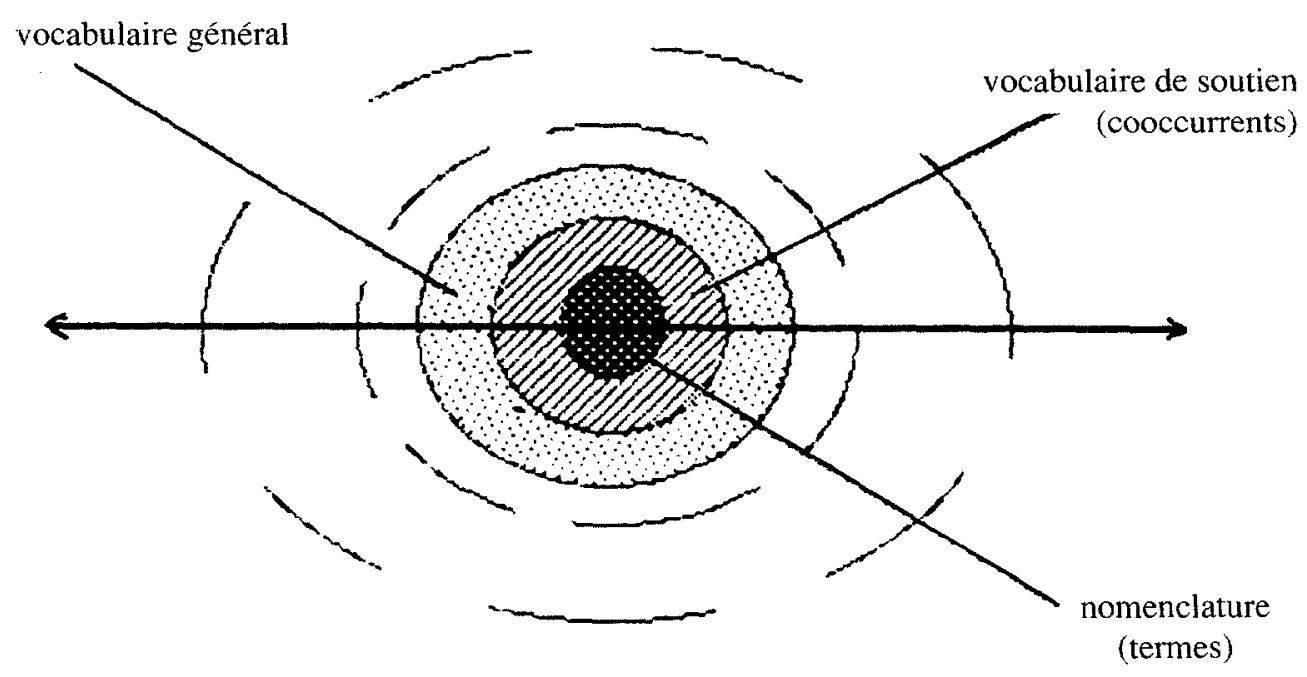

Formation du sens en langue de spécialité

À quoi il convient d'ajouter la stylistique, soit l'art d'agencer ces divers éléments de façon à en tirer certains effets - et, sans doute, sens - particuliers selon la destination du texte spécialisé et sa fonction première: information, connaissance, érudition ou savoir.

\section{LE DROIT ENVISAGÉ COMME LANGUE DE SPÉCIALITÉ}

La notion de LS revêt un sens tout à fait particulier dans le cas de la langue juridique, notamment en raison des fonctions sociales qui incombent à cette dernière. Sinon, en quoi celle-ci se distinguerait-elle des autres langues et des différents types de discours, ou même de la langue commune? Quels sont les paramètres susceptibles d'en fournir la définition la plus juste? Ses caractéristiques lexicales, syntaxiques et stylistiques sont-elles suffisantes pour lui attribuer une identité propre ou, au contraire, devrait-on tenir compte de variables relevant davantage de ses fonctions et de ses applications?

Ces questions complexes appellent des réponses nuancées et circonstanciées. Si le langage peut être défini comme un mode particulier d'expression, on ne peut nier que chaque domaine possède le sien, constitué d'usages, de tournures et de formules parfois singulières, quoiqu'ils procèdent néanmoins, le plus souvent, de la langue courante. Le fait de reconnaître ces usages et tournures comme caractéristiques du langage du droit permet-il d'affirmer pour autant (Sourioux et Lerat 1975) qu'ils constituent un ensemble d'éléments étrangers au système général de la langue? Ce qui reviendrait à postuler l'existence de «langues de spécialité» autonomes.

Si chacun ou presque - linguistes comme juristes - s'entend sur l'existence d'une terminologie essentiellement juridique (Gémar 1980:881), en revanche les avis 
sont très partagés sur la question de la spécificité de ces termes et de leur essence juridique. Pour les uns, praticiens généralement, les choses semblent aller de soi : « [...] la langue du juriste doit être extrêmement précise [...] ce qui n'est pas clair n'est pas juridique [...] il est nécessaire que les mots présentent des contours définis.» (Nerson 1967: 607). D'autres voient les choses avec davantage de circonspection. Pour Aurel David par exemple, le Droit, faute d'avoir développé une connaissance analytique encore à venir, en est réduit à la «pensée naïve» (David 1982: 33). Ou encore H.L.A. Hart: «[...] the great anomaly of legal language is our inability to define its crucial words in terms of ordinary factual counterparts.» (Hart 1953: 7-8). Ce jugement rejoindrait celui qu'Aurel David portait, voici une quinzaine d'années, sur le nombre de termes spécifiquement juridiques, très restreint. Après des années de recherche, il en était arrivé au nombre d'une cinquantaine seulement! Certains philosophes du droit, dont Michel Villey, vont même jusqu'à contester l'existence d'un langage scientifique du droit, dans la mesure où la société contemporaine a perdu le fil d'une définition spécifique de l'objet voire même de la fin du droit. Contaminé par les divers courants de morale et les langages scientifiques et techniques successifs, le vocabulaire fondamental du droit se serait délité au point d'avoir perdu sa signification première (Villey $1975: 222$ ).

Or, comme chacun sait, il n'est guère aisé de dissocier, autrement que pour en faire l'analyse clinique, le lexique de la syntaxe, ni celle-ci de la stylistique, ni les deux premiers de la troisième, car l'un est imbriqué dans l'autre et ils réapparaissent, en situation, dans le suivant. De la même façon, le fond et la forme sont inextricablement mêlés dans le texte réalisé, juridique ou autre, au point d'en constituer le fondement même et de ne pouvoir en être exclus, sinon au risque de faire éclater la cohésion interne du texte. Le message de l'exemple donné plus haut, en clair, équivaut à ceci: on ne commet pas un délit impunément. Il pourrait être formulé de bien des façons. Toutes reviendraient à dire (à peu près) la même chose, que l'on dise :

Nul ne doit commettre de délit, à peine de sanction.

Toute personne qui commet un délit en subira les conséquences.

Celui qui commet un délit s'expose à des sanctions.

De délit ne commettras, car à sanction t'exposeras.

Si tu commets un délit, tu seras puni.

Qui un délit a commis, s'en trouvera marri.

etc.

La formulation est affaire de style, donc de goût personnel, mais avant tout de niveau de langue, de texte et de communication. Une des caractéristiques du langage du droit est son niveau d'expression: c'est l'État qui parle, du moins le plus généralement (législateur, juge). À l'évidence, la technicité propre aux langages techniques caractérise également le langage du droit. Avant même de buter sur la complexité du cheminement de la pensée juridique ou de l'articulation du texte, le profane est mis en présence d'un langage souvent hermétique empreint de concepts complexes dont l'articulation accentue l'opacité. En fait, il lit des mots dont il ne comprend pas le sens ou, pis, dont il s'imagine comprendre la signification. Tel est le mystère - et le risque - de la sémantique.

\section{SENS ET SIGNIFICATION}

Le problème de la sémantique est fondamental dès que l'on aborde la notion de langue et de texte, juridique ou autre. En droit toutefois le problème s'aggrave du fait que le texte juridique véhicule une norme généralement contraignante, comminatoire parfois. Il s'ensuit que le sens de ce texte et son interprétation sont potentiellement lourds de 
conséquences, car ils sont porteurs d'effets juridiques susceptibles d'être dommageables pour les personnes ou les parties concernées. Or ce qui vaut pour une personne a valeur universelle.

Par ailleurs, chercher à éclaircir le mystère du sens caché derrière les mots, la langue et le texte, en droit comme ailleurs, représente une tâche comparable aux travaux d'Hercule ou à la quête du Graal. Nous sommes entourés de signes porteurs de sens et de significations que nous appréhendons par le canal des cinq sens, et parfois ... du sixième, dont la Nature nous a dotés. Mais nous sormmes incapables de dire comment, parce que nous ignorons tout des mécanismes permettant à notre intelligence de comprendre ou saisir le sens des interrogations qui nous sollicitent sans cesse, à tout propos (Changeux 1983). La pensée échappe encore au processus d'explication que l'on peut tenter de proposer, d'un texte par exemple.

La linguistique classique avance une esquisse de solution en postulant une distinction fondamentale entre le sens et la signification (Hagège 1985: 215). Le sens serait un phénomène propre au texte comme combinaison de signes dans une situation donnée de parole, alors que la signification serait la caractéristique du signe.

Cette solution est intéressante et utile dans la mesure où elle reste circonscrite au contexte linguistique, celui du texte, celui du signe (linguistique). Mais elle est remarquablement insuffisante lorsqu'il s'agit de proposer une esquisse de théorie générale du sens. La linguistique n'y constituerait alors qu'un des éléments nécessaires et indispensables, certes, pour hasarder une explication, mais néanmoins insuffisants pour rendre crédible une solution qui ne tiendrait pas compte des innombrables facteurs intervenant dans le processus de réalisation du sens. J'entends par là non la prise de sens superficielle que l'on peut faire à la simple lecture d'un texte, le déchiffrage des signes, mais le sens profond, celui que Barthes qualifie de signifiance et qui peut aller jusqu'à «l'illumination» de Poincaré. Pour atteindre ce niveau extrême de saisie du sens il faut sortir du contexte strictement linguistique (les mots) pour aller aux «choses» et faire intervenir une réflexion épistémologique fondée aussi sur les sciences dites exactes et non uniquement sur la spéculation.

Si l'univers est régi par des lois irréfutablement établies par les physiciens, on voit mal pourquoi, en vertu de quelle logique humaine plutôt que scientifique, l'infiniment petit, par exemple le sens d'un mot/terme ou d'un texte, échapperait aux lois universelles de l'infiniment grand et ne serait pas régi par des forces ou interactions dont nous n'avons que peu ou pas conscience, mais qui n'en sont pas moins à l'œuvre dans le processus de production du langage, et donc du texte.

À partir d'ici, la linguistique ne peut plus, à elle seule, fournir les données et les solutions nécessaires et suffisantes pour appréhender le phénomène du sens et tenter de le comprendre, voire de le décrire. Nous sommes entourés de signes porteurs de sens universel qui, pour être perçus, n'ont pas à passer par le canal des signes linguistiques. Nos sens, même s'ils se sont considérablement affaiblis depuis l'époque des cavernes, reconnaissent infailliblement bruits, mouvements, couleurs, matières, etc. sans aucune médiation linguistique. Le sens de ces manifestations naturelles ou physiques est universel puisqu'il caractérise une «chose» qui s'exprime et est reconnue par l'Homme (et, sans doute, par la plupart des animaux) en dehors de tout contexte langagier. Il s'agit alors d'un sens premier ou absolu, commun à tous: d'un commun dénominateur, mais le plus grand. C'est le niveau informationnel, celui auquel nous permet d'accéder la vision quand on constate que, puisque les feuilles d'un arbre bougent, il doit faire du vent; l'ouie, quand on entend un coup de tonnerre et que l'on peut en déduire que l'orage menace; le toucher, lorsque les premières gouttes de pluie tombent sur notre main; etc. De l'information on passe à la connaissance lorsqu'on ne se contente plus de s'en 
remettre à ses sens pour savoir le temps qu'il fait et que l'on consulte un thermomètre, un baromètre ou un pluviomètre pour connaître avec précision la température, la pression ou la quantité d'eau de pluie accumulée.

Il s'ensuit que le sens et l'interprétation qu'en fera une personne ne dépendent qu'en partie de la «lecture» des signes (sonores ou écrits) composant un texte puisque cette lecture nous est aussi personnelle que notre façon de marcher, d'écrire ou de parler. L'interprétation d'un texte est donc unique, comme nous le pensions intuitivement. Cette impression semble confirmée par les résultats des travaux les plus récents des neurologues et spécialistes du cerveau, pour lesquels le cerveau humain se développerait de façon unique et originale chez chaque individu, de sorte qu'il n'y en aurait pas deux qui soient identiques.

Nous aurions donc trois niveaux de sens qui, en se greffant les uns aux autres, produiraient les données fondamentales suffisantes pour qu'un lecteur - car il est surtout question ici de textes écrits - parvienne, à partir du texte proposé, à en retrouver le sens, avant d'en arriver à la signification, stade final de son processus d'interprétation. Il y aurait donc deux étapes dans ce processus qui seraient extralinguistiques, soit la première et la dernière. La première, nous l'avons vu, est celle du sens universel. La demière est celle qui renvoie à des connaissances préalables, faute desquelles le lecteur ne pourrait accéder à la signification. Entre les deux, se situent le prédicat «libre» et le prédicat «lié». Le sens du prédicat libre est celui, objectif, que recensent les dictionnaires et que toute langue possède en équivalence (Ex: maison = Haus, house, casa; droit = derecho, direito, diritto, Recht, Law). Le sens du prédicat lié sera celui, subjectif, que lui donnera le contexte particulier dans lequel il est situé: telle maison, à tel endroit; tel système de droit, dans tel pays. $\grave{A}$ mesure que le lecteur progresse vers la signification du texte, le dénominateur commun se réduit. D'universel au départ, il tend vers le particulier, à l'arrivée. La signification sera, selon le degré d'aptitude du lecteur, le plus petit commun dénominateur, car tout le monde n'y accède pas également (et, pour un traducteur, ne la rend pas identiquement). Autrement dit, si l'on comparait le sens à un angle, il serait plat au début et aigu à la fin.

C'est finalement au terme de ce parcours que le lecteur, après avoir redonné vie, donc sens au texte qu'il lit - quel qu'en soit le motif: simple lecture, traduction, recherche, plaisir ou travail - à la suite de cet échange à la fois muet et prolixe, parvient à la signification. Que celle-ci soit juste ou déviante, approximative ou faussée ne change rien à la chose. La valeur du résultat est un autre problème, celui du niveau du lecteur, de sa capacité à comprendre ce qu'il lit, et donc à l'interpréter correctement.

Nous avons vu le schéma général. Appliqué à un domaine particulier ressortissant de surcroît aux sciences sociales comme celui du droit, que peut apporter un tel schéma universel?

\section{SENS ET SIGNIFICATION DU DISCOURS JURIDIQUE}

Le langage du droit n'est qu'un sous-ensemble de l'ensemble que constitue la langue. Lui-même système particulier régi par ses propres lois internes, il fait néanmoins partie du système linguistique général, dont il ne peut s'abstraire. Ce fait ne peut être nié: le droit ne saurait exister sans la langue, (alors que la langue pourrait très facilement se passer du droit!). G.L. Williams nous rappelle à cette évidence: «[...] the law is only a special department of language [...] whereas application of law is limited, the language is all pervasive [...]» (Williams 1945:73).

Ensuite, l'idée même de «langage du droit» pourrait induire en erreur le lecteur non averti en lui laissant croire que le droit s'exprime en bloc, d'une seule et même voix. Or, comme tout discours spécialisé, le droit est polymorphe et polyphone. Il emprunte, pour 
exprimer son message, de très nombreuses voies et revêt d'innombrables formes. La typologie des textes juridiques sommairement esquissée ailleurs (Gémar 1987: 504) en administre la preuve. Le texte juridique possède quatre formes principales d'expression, chacune d'elles étant subdivisible à son tour en un nombre $\mathbf{n}$ de sous-ensembles. En fait, à la limite, on peut avancer qu'il y a autant de types de textes qu'il y a de situations juridiques. Quelle commune mesure y a-t-il, en effet, entre le texte de la constitution d'un pays, loi suprême réglant la marche des ses institutions, et une loi sur l'éclairage des rues de telle ville par becs de gaz? En outre, le législateur, le juge, le notaire ne s'expriment pas de la même manière, ne s'adressent pas aux mêmes personnes (citoyens, justiciables, particuliers). Enfin, en droit comme dans les autres domaines, un texte peut être informationnel, technique, savant ou érudit plutôt que seulement pragmatique ou esthétique. Selon le cas, ses modes d'expression en seront conditionnés et le texte sera porteur d'énonciations représentatives, directives, consensuelles, expressives, déclaratoires, etc. (Danet 1980). En fonction de quoi, la stylistique, la syntaxe et le lexique du texte juridique varieront de façon non négligeable. Quant à la sémantique, elle suivra la fonction première du texte et, comme dans l'aspect général vu plus haut, elle s'articulera de la même manière que pour toute autre langue de spécialité: du sens à la signification. L'interprétation de cette signification est d'ailleurs une des tâches les plus délicates dont est investi le juriste, principal interprète du langage du droit.

L'élément fondamental ou premier de sens, nous l'avons vu, sourd du terme, véhicule principal des notions d'un domaine. Mais pour prendre vie, ce terme a besoin de s'appuyer sur un vocabulaire quasi juridique (le vocabulaire de soutien/cooccurrents) et, enfin, général. L'acte interprétatif du sens, puis de la signification suivra ces trois étapes, chez tout lecteur, la compréhension grossière étant le fait du profane, la fine restant l'apanage du spécialiste. Entre les deux s'étend, en théorie, toute la gamme des interprétations possibles ...

Au sommet de la hiérarchie juridique, le législateur énonce le sens du langage du droit, mais c'est le juge qui en fixe la signification lorsqu'il est appelé à interpréter le texte litigieux qui lui est soumis par les parties. Que fait alors cet interprète du droit? Il revient au sens ordinaire des mots en consultant les dictionnaires généraux! Cette démarche tendrait à prouver que le langage du droit, en définitive, ne diffère guère de la langue commune, voire qu'il se confond avec elle, du moins pour ce qui est des mots du vocabulaire de soutien et du vocabulaire général, soit la très grande majorité du lexique d'une langue, contre une petite minorité de termes techniques.

À propos de la technicité des langues de spécialité qui équivaut dans la bouche des spécialistes du langage à l'univocité du terme technique, là encore il faut distinguer, à l'instar de Piaget, ce qui est d'ordre nomothétique (= universel) de ce qui ne l'est pas. Selon lui, les sciences juridiques ne seraient pas nomothétiques, contrairement à la sociologie, la linguistique, l'économie ou la démographie (Piaget 1964: 601). N'ayant pas pour vocation de dégager des lois, les sciences juridiques «constituent un monde à part, dominé par des problèmes, non pas de faits ou d'explications causales, mais de normes.» (p. 601). Or, comme chacun sait, la norme est mouvante, instable par définition, surtout quand elle est d'ordre social. Le vocabulaire juridique reflète cette instabilité, quoi qu'en pensent les juristes. Il en découle une polysémie qui contribue à épaissir la trame du mystère dont est entouré le droit et qui découle en grande partie de son caractère solennel et ésotérique (naissance en assemblée législative; sanction publique, par la Justice, de l'infraction commise; célébration du Droit par les ministres du culte que sont avocats et notaires).

Cette polysémie, plus ou moins prononcée selon les domaines, est inhérente au langage humain. Elle se cache d'ailleurs derrière les termes parfois les plus anodins. Par 
exemple, prenons un mot tout à fait banal comme l'anglais information. Replacé dans le contexte de l'article 785 du Code criminel du Canada, il ne signifie plus la banale «information» ou les «renseignements» que tout lecteur s'attendrait à y trouver, mais une... «dénonciation»! Et que dire d'un terme aussi répandu que business corporation. Au Canada, il n'a pas moins de 6 équivalents possibles selon qu'il sera traduit en français au Québec, en Ontario, au Nouveau-Brunswick ou à Ottawa, que le texte sera antérieur ou postérieur à 1985! Ce ne sont que deux exemples entre mille. Le droit abonde en termes apparemment «communs» ayant un sens technique néanmoins très précis. Cette particularité, soit dit en passant, doit rendre le traducteur doublement prudent lorsqu'il a affaire à un texte juridique. Rappelons à ce propos ce principe cardinal de Lord Halsbury: «The more words there are, the more words are there about which doubts may be entertained.» (Halsbury 1907).

En somme, si l'on part du postulat que, pour «signifier», le texte juridique doit remonter jusqu'aux tribunaux, où les juges lui donneront son interprétation finale, donc sa véritable signification, force est d'admettre que le langage du droit n'est jamais fixé et que la langue juridique est d'essence éphémère, incertaine. Le sens de ses termes est circonscrit, mais leur signification n'est établie que dans une situation donnée, très précise, appliquée à un cas en particulier. Autrement dit, le langage du droit n'est jamais (ou presque) achevé, c'est un langage se faisant, en voie de réalisation permanente, qui ne se matérialise que dans une infime minorité de cas par rapport à l'ensemble des textes innombrables - produits qui ne sont pas soumis à l'interprétation des tribunaux. De ce fait, il ne se différencie guère du langage ordinaire, en évolution sémantique constante, sinon par le fait que son évolution est plus lente, quoique inéluctable.

\section{CONCLUSION}

C'est ainsi que le cycle du sens et de la signification s'accomplit. Cycle est bien le mot qui le décrit le mieux, en tout cas pour ce qui est des langues de spécialité, puisque du terme (particulier) on arrive petit à petit au discours (général) mais que l'on rejoint de nouveau le particulier au terme du processus de signification, celle-ci n'étant que le sens spécial que prendra le texte dans le contexte singulier où il s'inscrit. Le phénomène sémantique est matérialisé par le signe linguistique, lequel le cristallise en quelque sorte, mais ce demier n'en est que le contenant. Le contenu, c'est le lecteur qui l'extrait du texte par son interprétation. Ce faisant, il lui (re)donne vie par l'effet d'une action que l'on peut difficilement qualifier autrement que «thermodynamique». Échange il y a, en tout cas, au cours duquel l'information inerte enfouie dans le texte reprend vie et forme sous l'impulsion (la force?) du regard humain qui lui fera subir une métamorphose, plus ou moins complète et réussie selon les individus, dont sortira d'abord le sens et, peut-être, la signification. Des forces et des lois échappant encore à toute observation humaine sont manifestement à l'œuvre en l'espèce. La linguistique, lorsqu'il s'agit d'aborder la question du texte et de sa sémantique, n'est que le gros arbre derrière lequel s'abrite du regard la forêt immense que représente l'infiniment grand par rapport à l'infiniment petit. Cette inégalité n'est, après tout, que le reflet de la loi d'Heisenberg: les concepts humains étant impuissants à décrire les phénomènes (la réalité), l'incertitude demeure.

En définitive, comme l'univers, le langage vit, est en évolution permanente. Le langage du droit, qui est une des composantes du langage tout court, voire se confond avec lui, n'échappe pas à cette loi. Le juriste non plus. 


\section{BIBLIOGRAPHIE}

CHANGEUX, Jean-Pierre (1983): L' homme neuronal, Paris, Fayard.

DANET, Brenda (1980): «Language in the Legal Process», Law \& Society Review, 14-3, pp. 447-493.

DARBELNET, Jean (1979) : «Réflexions sur le discours juridique», Meta, 24-1, pp. 26-34.

DARBELNET, Jean (1982): «Niveaux et réalisations du discours juridique», Langage du droit et traduction, Montréal, Linguatech et Conseil de la langue française, pp. 51-60.

DAVID, Aurel (1982): «Les termes élémentaires du droit», Langage du droit et traduction, Montréal, Linguatech et Conseil de la langue française, pp. 31-38.

GÉMAR, J.-C. (1980): «La langue juridique, langue de spécialité au Québec : éléments de méthodologie», The French Review, 53-6, p. 880-893.

GÉMAR, J.-C. (1987): «La traduction juridique : art ou technique d'interprétation?», Revue générale de droit, Ottawa, Éditions de l'Université d'Ottawa, pp. 495-514.

HAGEGE, Claude (1985): L' homme de paroles, Paris, Fayard

HALSBURY (Lord) (1907): Laws of England, London, Butterworths.

HART, H.L.A. (1953): Definition and Theory in Jurisprudence, Oxford Clarendon Press [cité par Ethel Groffier, dans La lexicographie juridique, Cowansville (Québec), Yvon Blais, 1990, p.52.

NERSON, Roger (1967): «Exercices de vocabulaire», Mélanges Voirin, Paris, L,G.D.G., pp. 603-617.

PIAGET, Jean (1964) : «Classification des disciplines et connexions interdisciplinaires», Revue internationale des Sciences sociales, 16-4, pp. 598-616.

SOURIOUX, J.-L. et P. LERAT (1975) : Le langage du droit, Paris, PUF.

VILLEY, Michel (1975): Philosophie du droit. Définitions et fins du droit, Paris, Dalloz, coll. Précis Dalloz.

WILLIAMS, G.L. (1945): «Language and Law», Law Quarterly Review, 61. 